\title{
Decoupling Technique Using Ferrite-Film Loading for 5G MIMO Applications
}

\author{
Di Wu, ${ }^{1}$ Yang Qiu, ${ }^{1}$ Guoliang Yu, ${ }^{1}$ Rongdi Guo, ${ }^{1}$ Guohua Wu, ${ }^{1}$ Jiawei Wang, ${ }^{2}$ Yi Zhang, \\ Mingmin Zhu $\mathbb{D}^{1,4}$ and Hao-Miao Zhou $\mathbb{D}^{1}$ \\ ${ }^{1}$ Key Laboratory of Electromagnetic Wave Information Technology and Metrology of Zhejiang Province, \\ College of Information Engineering, China Jiliang University, Hangzhou 310018, China \\ ${ }^{2}$ College of Science, Zhejiang University of Technology, Hangzhou 310023, China \\ ${ }^{3}$ Key Laboratory of Wireless Power Transmission of Ministry of Education, College of Electronics and Information Engineering, \\ Sichuan University, Chengdu 610064, China \\ ${ }^{4}$ Center for X-Mechanics, Institute of Applied Mechanics, Zhejiang University, Hangzhou 310027, China
}

Correspondence should be addressed to Mingmin Zhu; mzhu@cjlu.edu.cn and Hao-Miao Zhou; zhouhm@cjlu.edu.cn

Received 19 October 2021; Revised 23 November 2021; Accepted 18 January 2022; Published 8 February 2022

Academic Editor: Ahmed Toaha Mobashsher

Copyright $(0) 2022$ Di Wu et al. This is an open access article distributed under the Creative Commons Attribution License, which permits unrestricted use, distribution, and reproduction in any medium, provided the original work is properly cited.

\begin{abstract}
Decreasing the mutual coupling between multiple-input-multiple-output (MIMO) antenna elements requires the selection of an appropriate decoupling technique without changing the geometry or operating frequency of the antenna. In this paper, a simple and flexible approach that involves loading a small ferrite film on the radiation patch of an MIMO antenna pair has been proposed to mitigate the unwanted mutual coupling. Through a thorough investigation, the two IFA elements with an element distance of only $1.4 \mathrm{~mm}$ can be decoupled adequately by properly controlling the ferrite-film loading area and the thickness of the ferrite film, along with maintaining the desired operating frequencies and acceptable radiation characteristics at the LTE Band 42 $(3.4-3.6 \mathrm{GHz})$. The optimum ferrite-film loading on the grounding branch of the IFA element can increase the isolation from $-6.64 \mathrm{~dB}$ to $-10.43 \mathrm{~dB}$ and enhance the bandwidth from $3.20-3.88 \mathrm{GHz}$ to $2.83-3.65 \mathrm{GHz}$, and its efficiency can reach to $45.5 \%$, as well as a $2 \mathrm{~dB}$ gain. A physical model has also been established to interpret the operating mechanism of ferrite-film loading on the IFAs. The proposed simulated procedure is validated via measurements. This technique of ferrite loading on MIMO antennas can effectively decouple the mutual coupling at a low cost and with low design complexity, indicating its potential applicability for use in MIMO antennas in mobile handsets.
\end{abstract}

\section{Introduction}

Since $5 \mathrm{G}$ communication has become a commercial reality, the demand for higher channel capacities and data rates has significantly increased in wireless communication systems $[1,2]$. The use of multiple-input-multiple-output (MIMO) technology is an effective way to enhance the spectrum efficiency and increase the channel capacity. This technology can allow a communication system to send and receive more than one data-signal stream simultaneously over the same radio channel by utilizing uncorrelated channel paths in a multipath environment [3]. However, the MIMO technology could suffer from the mutual coupling between the MIMO antenna elements, which could deteriorate their channel capacity $[4,5]$. Therefore, decoupling techniques are crucial to improve the efficiency and throughput of communication systems with a large number of antennas; however, this is challenging in mobile terminals, which have significant space constraints.

Recently, significant research has been conducted into mitigating unwanted mutual coupling using various decoupling techniques. In some studies [3,6-8], a neutralization line connecting two coupled radiation patches has been used to suppress the coupling current, which could improve the isolation. The defected ground structures and electromagnetic band-gap structures have been verified to be effective methods to change the surface wave or current flow paths, increasing the isolation [9-11]. A parasitic structure 
or a shared radiation patch has also been proposed as decoupling designs to achieve good isolation by offering an extra coupling path, if space permits [12-15]. In addition to these decoupled structure designs, the concept of field distributions has been used to optimize the relative position of the antennas to obtain low mutual coupling, including current distributions [16], weak field distributions [17], and null-amplitude field points [18]. In addition, metamaterial, metasurface, and substrate integrated waveguide (SIW) structures have also been used to suppress the coupling between neighboring antenna elements, which can be applicable for a wide range of frequency bands with ease of manufacture process, low cost, and high isolation level between the array elements [19-22]. Although all of the aforementioned decoupling methods perform well, they require additional decoupling structures or networks, which occupy extra space and make the MIMO antenna's structure not flexible for adjustment.

For industrial applications, high levels of isolation are required, using a single flexible design with miniaturization capabilities. The proposal of novel materials could potentially help alleviate the fundamental limitations facing antennas in terms of size [23]. Therefore, magnetodielectric materials-combining both dielectric and magnetic properties-have been widely investigated for their use in antennas to change their radiation properties. Among these magnetic materials, ferrites exhibit the ability to simply tune the operating characteristics of microstrip patch antennas, while keeping the design complexity and cost relatively low. Important goals when using ferrite materials in compact antenna applications are antenna miniaturization and bandwidth enhancement, which are crucial to facilitate sufficient separation between the two antennas in the limited space of MIMO systems [24-29]. However, ferrites have low electrical conductivity, which decreases the eddy current losses, making them ideal components for high-frequency devices, such as isolators, circulators, phase shifters, and magneto-optical devices [30-34]. However, this isolation effect has rarely been considered in antenna design, which could benefit the control of interacting electric current distribution on the MIMO antennas.

In this paper, a simple method is proposed that involves loading a small piece of microwave ferrite film on the antenna elements to mitigate unwanted mutual coupling and realize a compact 5G mobile MIMO system design. The proposed MIMO antenna, based on inverted-F antenna (IFA) elements, operates in the $3.5 \mathrm{GHz}$ band $(3.4-3.6 \mathrm{GHz}$, LTE Band 42), which is one of the sub- $6 \mathrm{GHz}$ bands. In the two-element MIMO antenna design, the effects of the ferrite-film loading area and thickness on the radiation and isolation characteristics have been systematically investigated through both simulations and experimental studies. Through the proper control of ferrite-film loading, the two IFAs become decoupled, with high levels of isolation. The operating mechanism of ferrite-film loading on the decoupling of MIMO antennas is described in detail. Finally, the two-IFAbased MIMO antenna has been fabricated and tested to verify that the ferrite-film decoupling concept works well in the 3.5 $\mathrm{GHz}$ band of $5 \mathrm{G}$ mobile MIMO terminals.

\section{Antenna Design and Performance}

First, we would like to introduce the basic physical principle of the ferrite-film loading-based decoupling method. The schematic of the base MIMO antenna without considering any decoupling technique is shown in Figure 1(a), using two IFAs to provide the target operating frequency of LTE Band $42(3.4-3.6 \mathrm{GHz})$ for $5 \mathrm{G}$ smartphones. The nearest edge-toedge distance is only $1.4 \mathrm{~mm}$ (about $0.017 \lambda$ at $3.60 \mathrm{GHz}$ ). The two back-to-back monopoles are placed on the long edge of the top layer of the $1 \mathrm{~mm}$-thick FR4 substrate (the dielectric constant is 4.4 , and the tangent loss is 0.02 ), with an area of $24 \mathrm{~mm} \times 5 \mathrm{~mm}$, which are symmetrical along the center of the long edge. The metal ground is printed on the bottom layer of the substrate, with dimensions of $168 \mathrm{~mm} \times 66 \mathrm{~mm}$. Through optimization using the ANSOFT HFSS software, the simulated $S$-parameter diagram of the base antenna was determined, as shown in Figure 1(b), where Port1 is set as the excitation source and Port 2 is set to a $50 \Omega$ matching load. It can be seen that the resonant frequency is around $3.6 \mathrm{GHz}$ and the maximum depth of the return loss $\left(S_{11}\right)$ is $37 \mathrm{~dB}$, fully covering LTE Band $42(3.40-3.60 \mathrm{GHz})$, with a $-6 \mathrm{~dB}$ bandwidth. However, the isolation $\left(S_{21}\right)$ for the base antenna is higher than $-10 \mathrm{~dB}$ over LTE Band 42 , exhibiting strong mutual coupling between the two monopole elements. This coupling between the two radiation elements during normal operation is derived from the mutual interference effect, with the antenna distance being less than $0.28 \lambda$. An element distance of $0.28 \lambda$ is the critical distance for the overlap and nonoverlap of the element's effective area shapes and the element-gain paradox effect [35].

To understand the contributions of ferrite-film loading, the simulated surface current distributions of the proposed antenna at $3.6 \mathrm{GHz}$-without and with ferrite-film loading-are illustrated in Figure 2. These current distributions are obtained under the condition that Port1 is excited while Port2 is terminated to a matching load, and highly concentrated current distributions can be observed at the excited IFA under all conditions. On the base antenna without ferrite-film loading, a strong induced current in the loaded IFA can be clearly observed in Figure 2(a), implying strong mutual coupling in this two-element MIMO antenna. To verify the effect of ferrite-film loading on the decoupling phenomenon, three strong radiation areas have been selected on the excited IFA to load a $0.6-\mathrm{mm}$-thick ferrite film, as shown in Figures 2(b)-2(d). The ferrite material parameters used in the simulation are set as follows: relative permeability $\mu_{r}=100$, relative permittivity $\varepsilon_{r}=13$, and saturation magnetization $M_{S}=2500$ Gauss. With ferrite-film loading on different areas, the induced currents on the loaded IFA could be suppressed, leading to low mutual coupling. Among the loading areas, the most significant decrease in the current distribution on the loaded IFA occurs when the ferrite-film loading is on Area3, which exhibits the lowest mutual coupling. Therefore, we will compare the effects of the ferrite-film loading areas on the $S$-parameters to further demonstrate the effectiveness of the decoupling.

In practice, all elements in the MIMO antenna should be excited to improve the channel capacity, so we have loaded 


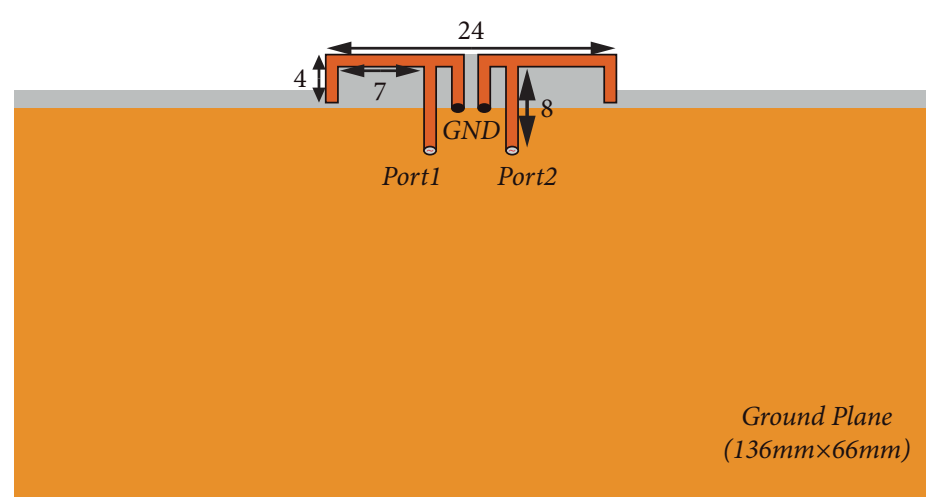

(a)

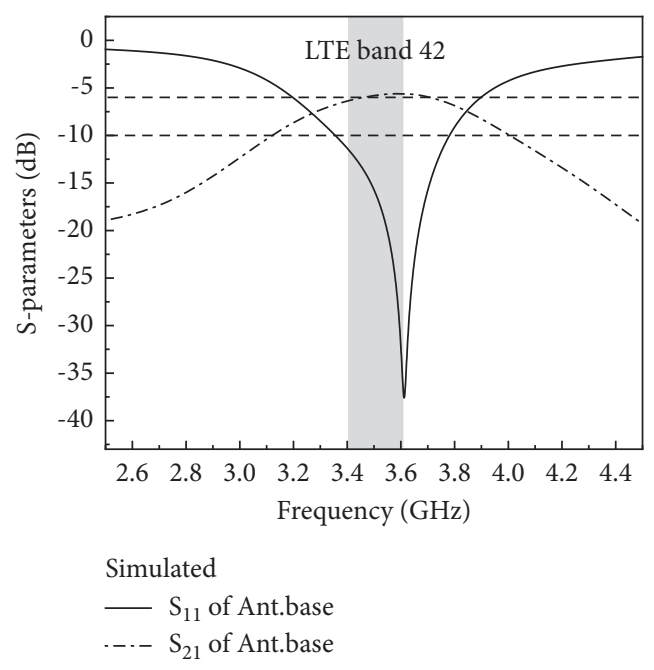

(b)

Figure 1: (a) Geometry and (b) simulated $S$-parameters of the proposed base antenna (unit: mm).

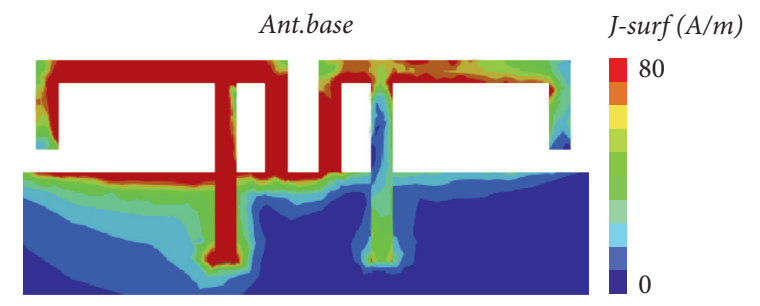

(a)

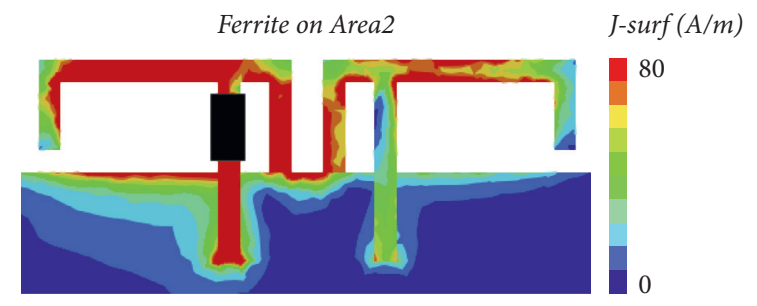

(c)

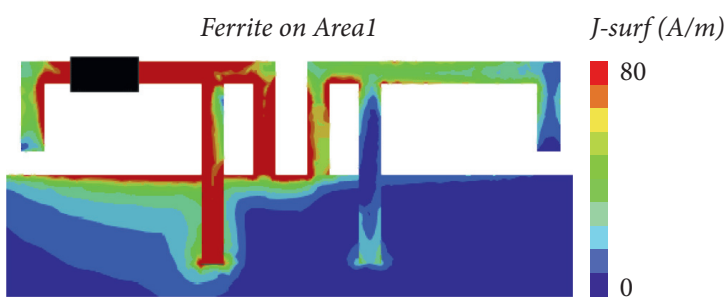

(b)

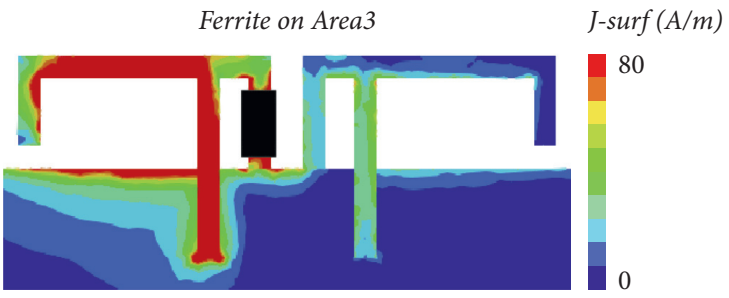

(d)

FIGURE 2: Surface current distributions of the proposed antenna (a) without ferrite, (b) with ferrite on Area1, (c) with ferrite on Area2, and (d) with ferrite on Area3.

the ferrite film on the symmetrical area of the two-element MIMO antenna, and the dual-antenna systems loaded with different ferrite film areas that have been optimized in Figure 2, defined as Ant.1, Ant.2, and Ant.3, are shown in Figure 3(a). When ferrite-film loading is conducted, the mutual coupling is significantly decreased over the entire frequency band. However, ferrite-film loading on different areas had differing $S$-parameters, as shown in Figures 3(b)3(d). For the ferrite-film loading on Area1, shown in Figure 3(b), the isolation of Ant.1, between Port1 and Port2, is better than $10.4 \mathrm{~dB}$ across $3.4-3.6 \mathrm{GHz}$, but the reflection coefficient decreases from $37.6 \mathrm{~dB}$ to $12.37 \mathrm{~dB}$ across the entire operating band. When Area2 is loaded with the ferrite film, as shown in Figure 3(c), the peak isolation of Ant.2 is higher than $-10 \mathrm{~dB}$ across the operating band, which indicates stronger mutual coupling than that of Ant.1. The reflection coefficient does not change drastically, but a $100 \mathrm{MHz}$ increase in the resonant frequency can be seen-from $3.60 \mathrm{GHz}$ to $3.70 \mathrm{GHz}$-with increases in ferrite-film loading. Finally, as shown in Figure 3(d), the ferrite film of Ant.3 covers Area3, and the simulated $S$-parameters, in this case, show good isolation characteristics-better than $10.61 \mathrm{~dB}$ across LTE Band 42 -implying a weaker mutual coupling than that of Ant.1. The reflection coefficient exhibits no obvious deterioration in the entire operating range. In addition, the resonant frequency decreases from $3.60 \mathrm{GHz}$ to $3.34 \mathrm{GHz}$ with increases in ferrite-film loading, exhibiting a different tendency than Ant.2. The operating mechanism underlying the effect of different ferrite-film loading locations on the IFAs will be further discussed by establishing corresponding physical models. 

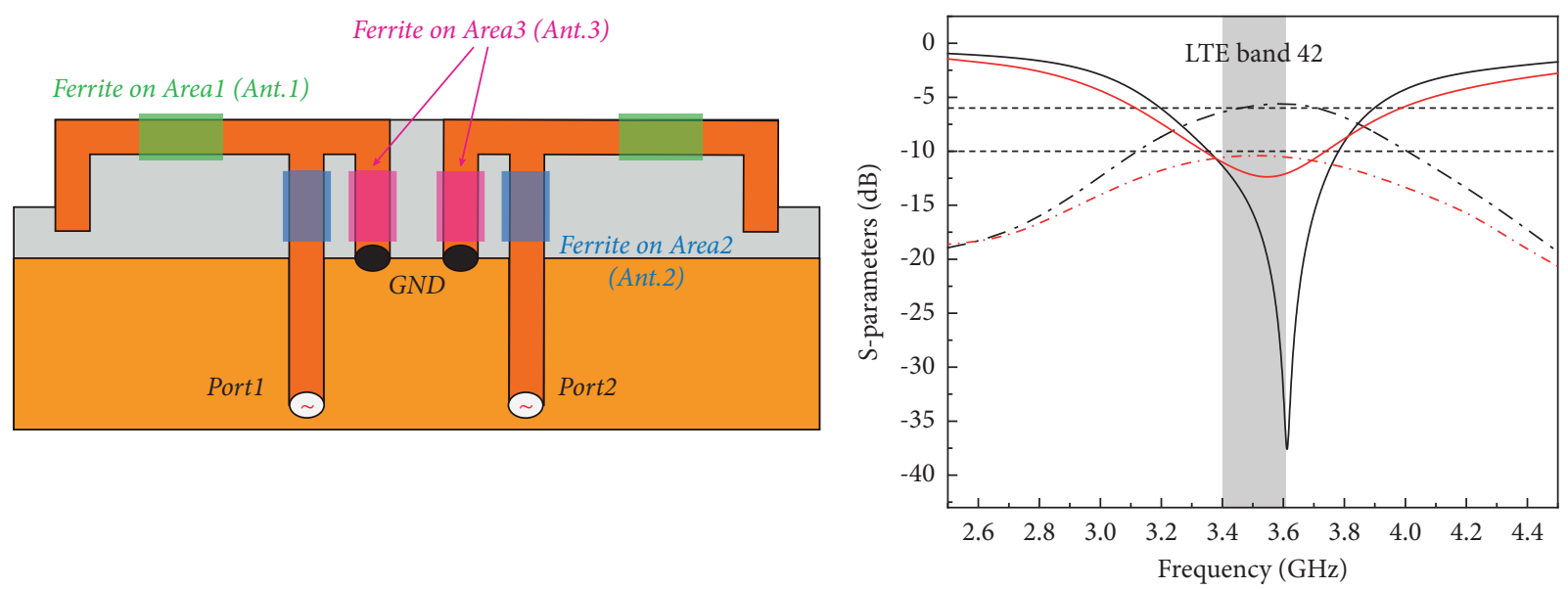

Simulated

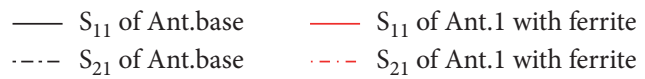

(a)

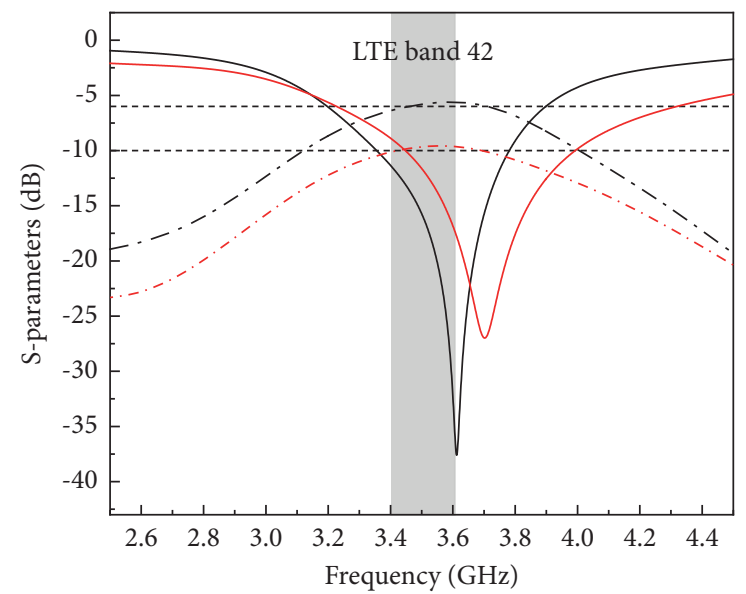

Simulated

$\begin{array}{ll}- & S_{11} \text { of Ant.base } \quad-S_{11} \text { of Ant.2 with ferrite } \\ \ldots- & S_{21} \text { of Ant.base } \quad \ldots-S_{21} \text { of Ant.2 with ferrite }\end{array}$

(c) (b)

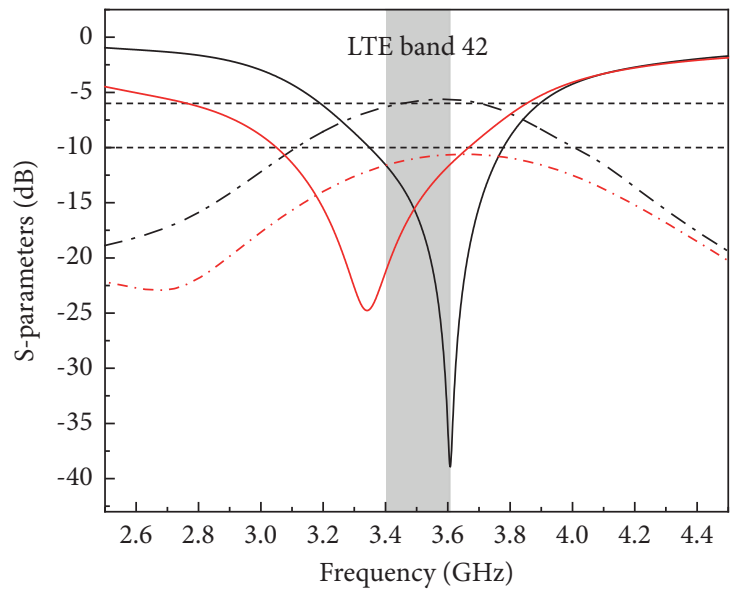

Simulated

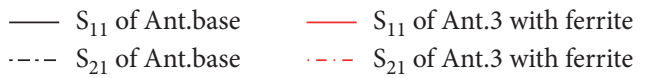

(d)

Figure 3: (a) 2D diagram of the proposed antenna with ferrite-film loading. Simulated $S$-parameters of (b) Ant.1, (c) Ant.2, and (d) Ant.3.

\section{Decoupling Analysis of Ferrite-Film Loading}

A model of a radiation equivalent circuit for the proposed antennas has been established to determine the effects of ferrite-film loading on the reflection coefficients of the antenna. The variations in the simulated impedance with different ferrite-film loading areas have been studied in Figure 4. According to the impedance theory in circuit analysis [36], the matching impedance of the antenna can be expressed as a complex number:

$$
Z=R+j X
$$

where $R$ is the equivalent resistance of the antenna and $X$ is the equivalent reactance of the antenna. When $X>0$, the reactance is inductive; otherwise, it is capacitive. Table 1 summarizes the $Z$-parameters of the antennas with ferrite film on different areas at their resonant frequencies. The impedance of the base antenna without ferrite-film loading at $3.60 \mathrm{GHz}$ is $Z_{11}=43.52+24.69 j$. By loading the ferrite film on different areas, different self-impedance changes can be observed in these antennas at the resonant frequencies. With loading the ferrite film on Area1, the real part of the impedance for Ant.1 increases from $43.52 \Omega$ to $71.24 \Omega$ and its imaginary part exhibits a change from 24.69 to 11.98 . This means that the ferrite-film loading on Areal primarily increases the equivalent resistance of the antenna and there is a variation in the inductive characteristic. When the ferrite film is loaded on Area2, the real part of the impedance for Ant.2 changes from $43.52 \Omega$ to $61.83 \Omega$ and its imaginary part drops from 24.69 to 8.60 . An increase in the equivalent resistance and a reduction in the reactance can be observed in Ant.2, indicating a reduced inductive characteristic for the 


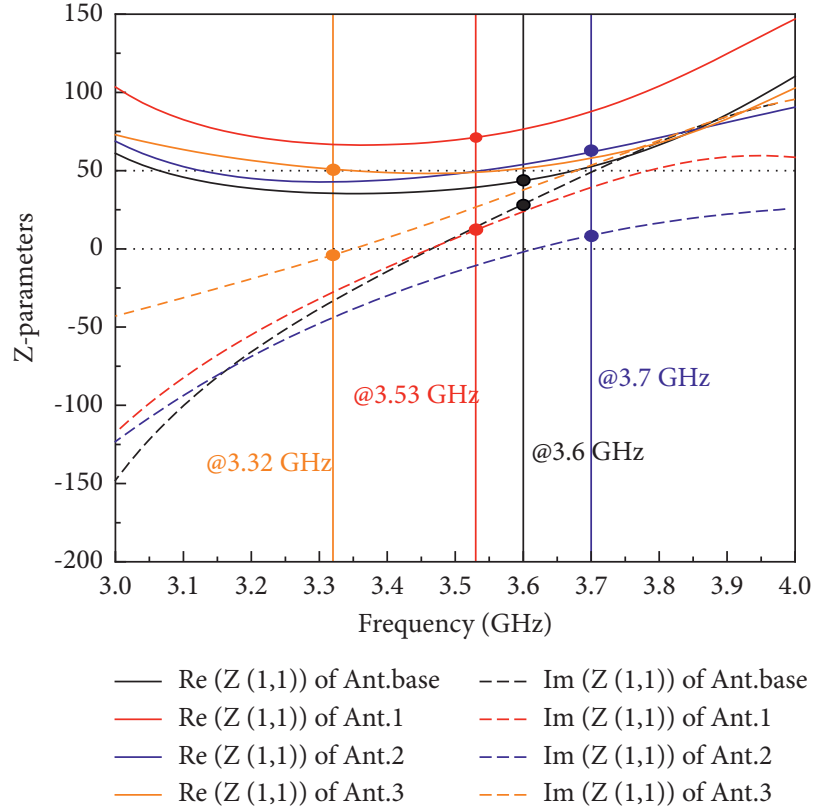

(a)

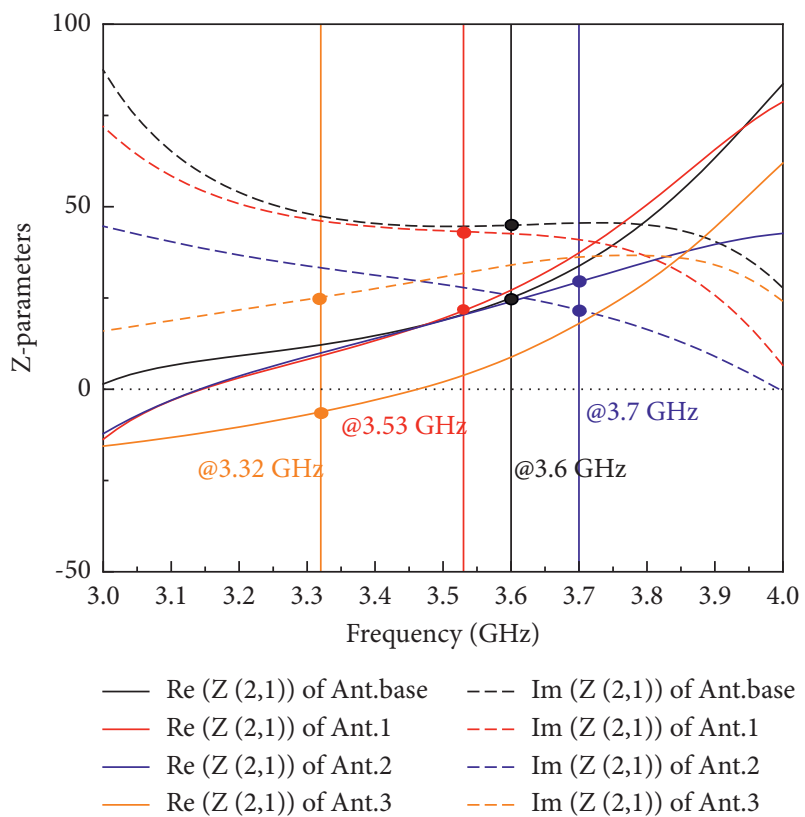

(b)

FIGURE 4: Impedance of (a) $Z_{11}$ and (b) $Z_{21}$ for the proposed antenna with different ferrite-film loading areas.

TABLE 1: Summary of $Z$-parameters for the antennas with ferrite film on different areas at the resonant frequencies.

\begin{tabular}{lcccc}
\hline & Ant. base & Ant.1 & Ant.2 & Ant.3 \\
\hline Frequency & $3.60 \mathrm{GHz}$ & $3.53 \mathrm{GHz}$ & $3.70 \mathrm{GHz}$ & $3.32 \mathrm{GHz}$ \\
$Z_{11}$ & $43.52+24.69 j$ & $71.24+11.98 j$ & $61.83+8.60 j$ & $50.9-3.92 j$ \\
$Z_{21}$ & $25+44.92 j$ & $21.51+43.18 j$ & $29.40+21.75 j$ & $-6.19+25.18 j$ \\
\hline
\end{tabular}

ferrite film on Area2. On the other hand, when the ferrite film is loaded on Area3, the real part of the antenna impedance rises from $43.52 \Omega$ to $50.90 \Omega$ and its imaginary part decreases from 24.69 to -3.92 . Again, the equivalent resistance increases in this case, but a differing variation in reactance could lead to an increased capacitive characteristic of Ant.3.

The dual IFAs can be considered to be equivalent to an RLC network [37], as shown in Figure 5(a), and the different radiation branches of strip1 could exhibit different impedance characteristics with resistance $R_{1}$, inductance $L_{1}$, and capacitance $C_{1}$ on the RLC circuit. First, in the process of magnetization, the hysteresis behavior in the magnetic material will lead to an energy storage phenomenon equivalent to a magnetic capacity $\left(C_{f}\right)$ and its energy dissipation part for the magnetic losses is equivalent to a reluctance $\left(R_{f}\right)$; therefore, the ferrite film can be assumed as a resistance $R_{f}$ and a capacitance $C_{f}$ in series. In Ant.1, the variation of impedance is as shown in Figure 4(a), where the branch in Areal should be resistive in the RLC circuit; when the ferrite film is loaded, the equivalent $R_{f}$ and $C_{f}$ will be in series with $R_{1}$. Therefore, this ferrite-film loading will lead to an increase in resistance and capacitance, indicating its impedance-variation trend adequately. Using this equivalent model, we can also define the branch in Area2 and the branch in Area3 as being inductive and capacitive for their variations in impedance, shown in Figure 4(a). With $R_{f}$ and
$C_{f}$ in parallel with $L_{1}$, the inductance of Ant.2 will decrease with small increases in resistance. In addition, with $R_{f}$ and $C_{f}$ in parallel with $C_{1}$, the capacitance of Ant.3 will increase with slight increases in resistance. These tendencies agree well with the characteristics of the trend of impedance variation with ferrite-film loading. The resonant frequency of the microstrip antenna can be expressed as follows [5]:

$$
\text { Freq }=\frac{1}{2 \pi \sqrt{L \cdot C}},
$$

where $L$ and $C$ are the equivalent inductance and the equivalent capacitance, respectively. Using the equation, the original resonant frequency can be shifted by changing the antenna's reactance characteristics. For Ant.1, its equivalent resistance differs significantly from the matching impedance of $50 \Omega$, which leads to a worse reflection coefficient and a downward shift in the resonant frequency owing to its effect on the equivalent reactance. For Ant.2 and Ant.3, the matching impedance is near $50 \Omega$, but the equivalent reactances exhibit obvious variations. As a result, reflection-coefficient curves can be observed in Ant.2 and Ant.3, while the inductance decrease will drive the resonant frequency to move to the high-frequency range in Ant.2 and the capacitance increase will result in the resonant frequency of Ant.3 moving to a lower frequency range. This model can explain the variation in reflection coefficient caused by loading ferrite films on different areas on the base antenna, as shown in Figure 3. 


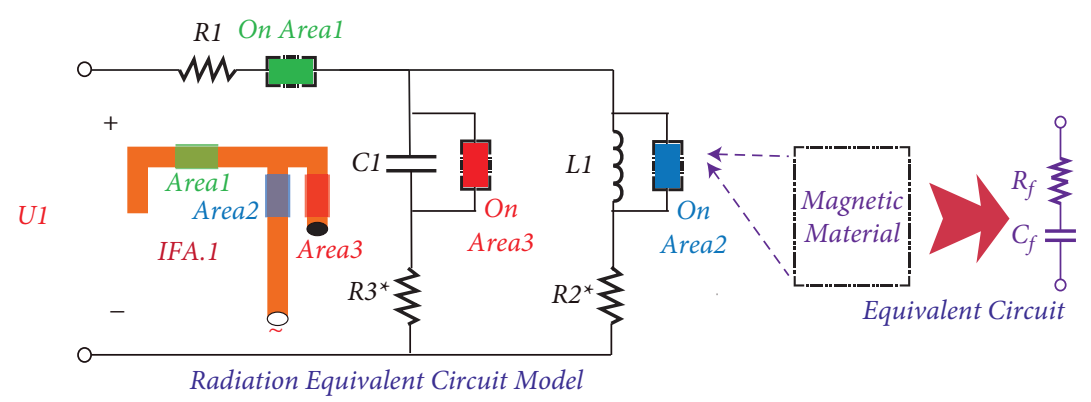

(a)

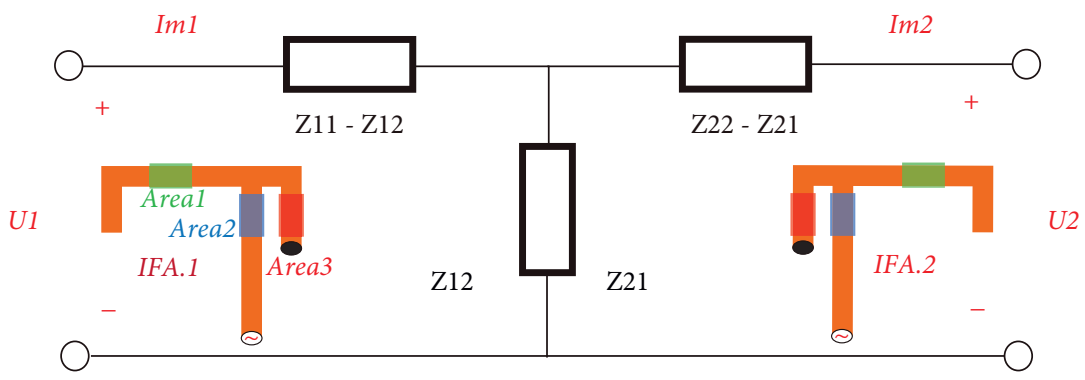

(b)

FIgURe 5: (a) Oversimplified radiation equivalent circuit model of the proposed antenna with ferrite-film loading. (b) Oversimplified coupled equivalent circuit model of the dual-element antenna.

The coupling between the dual-element antennas can be equivalent to a dual-port network, as shown in Figure 5(b). The radiation impedance of the symmetrical element can be expressed as follows:

$$
\begin{aligned}
Z_{\Sigma 1} & =\frac{U_{1}}{I_{m 1}} \\
& =Z_{11}+\frac{I_{m 2}}{I_{m 1}} Z_{12}, \\
Z_{\Sigma 2} & =\frac{U_{2}}{I_{m 2}} \\
& =Z_{22}+\frac{I_{m 1}}{I_{m 2}} Z_{21},
\end{aligned}
$$

where $Z_{11}, Z_{12}$, and $Z_{\Sigma 1}$ and $Z_{22}, Z_{21}$, and $Z_{\Sigma 2}$ are the selfimpedance, mutual impedance, and radiation impedance of IFA. 1 and IFA. 2 attributed to their respective antinode currents, respectively. With this formula, the smaller is the mutual impedance of the antenna, the smaller influence will be acted on the other antennas. As shown in Table 1, the mutual impedance of the base antenna without ferrite-film loading at $3.60 \mathrm{GHz}$ is $Z_{21}=25+44.92 j$. With loading the ferrite film on different areas, the mutual impedances of the three antennas at their resonant frequencies will be changed to $21.51+43.18 j, 29.40+21.75 j$, and $-6.19+25.18 j$, respectively. Obviously, the mutual impedance of Ant.3 shows the smallest value, indicating that the best isolation between the two IFAs will be achieved by loading the ferrite film on Area3.

To offer further insight into the decoupling performance, the surface current distributions of the proposed dual- element antenna with different ferrite-film loading areas were simulated; the results for which are compared in Figure 6. For the base antenna without ferrite-film loading, a strong induced current in IFA.2 can be clearly observed in Figure 6(a). On the other hand, with ferrite-film loading on different areas-as defined for Ant.1, Ant.2, and Ant.3 above-a significant reduction in current density can be observed in IFA.2. These observations imply that considerable isolation between the two elements can be obtained through ferrite-film loading.

The simulated $3 \mathrm{D}$ radiation patterns of the dual-element antenna with different ferrite-film loading areas are shown in Figure 7 when both Port 1 and Port 2 are fed at the same time. It can be seen that the ferrite coverage does not significantly change the original gain direction, but it makes the gain value decrease in all directions. Ant.1, with ferrite-film loading on Area1, exhibits an apparent degradation in the radiation pattern performance in comparison to that of the base antenna; however, there are only slight decreases in the radiation pattern when loading ferrite films on Area2 and Area3. This is consistent with the fact that the high magnetic loss of ferrite will lead to a low antenna gain [23, 38].

The effects of different ferrite-film thicknesses on the $S$-parameters of the three antennas with different loading areas are also simulated, and the results are shown in Figure 8. By increasing the thickness of ferrite film, the peak isolations of all antennas can be decreased to below $-10 \mathrm{~dB}$ within the desired operating band, showing good decoupling effect. However, different ferrite-film loading areas and different ferrite-film thicknesses will have different effects on the return loss characteristics. By loading ferrite film on Area1, the resonant depth of $S_{11}$ will change sharply with increasing the ferrite-film thickness, but with 


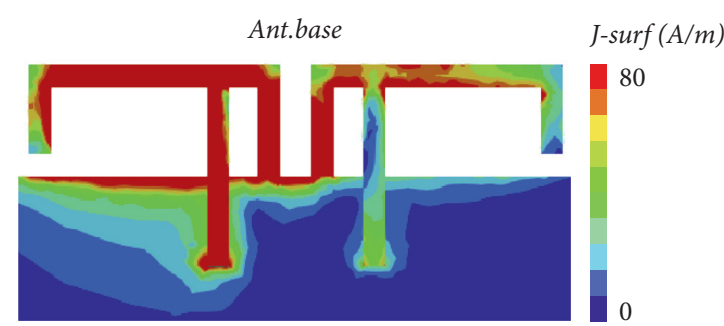

(a)

Ant.2

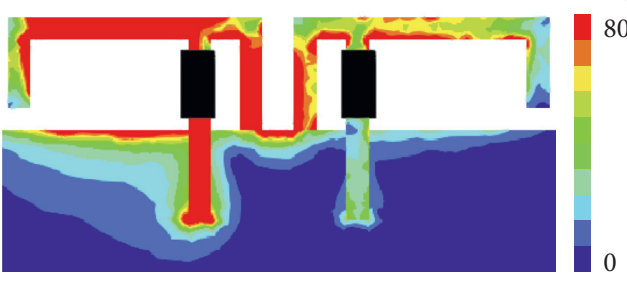

(c)

$J-\operatorname{surf}(A / m)$

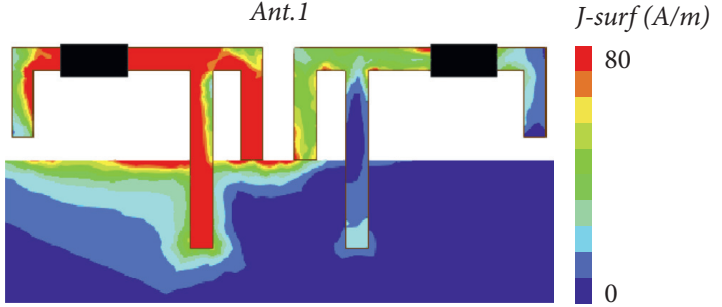

(b)

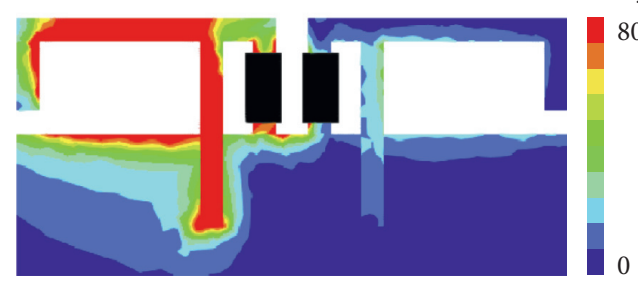

(d)

Figure 6: Surface current distributions of (a) the base antenna, (b) Ant.1, (c) Ant.2, and (d) Ant.3.

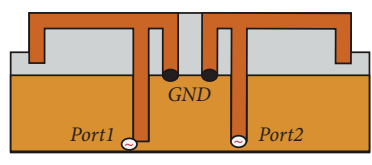

Ant.base

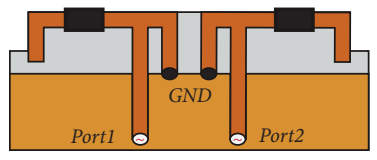

Ant.1

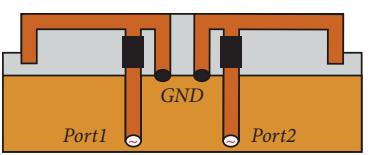

Ant.2

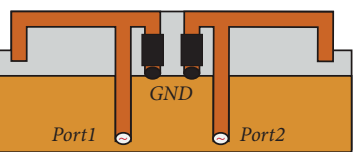

Ant.3

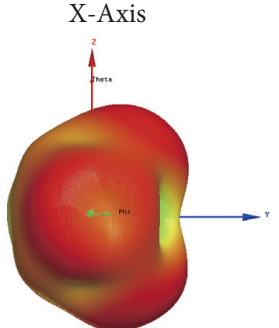

(a)

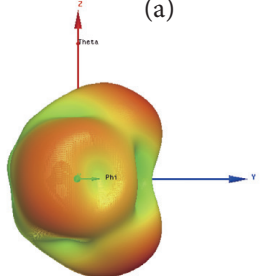

(d)

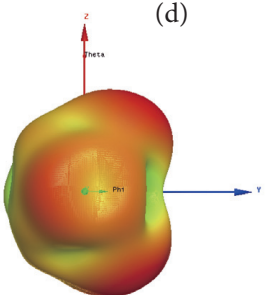

(g)

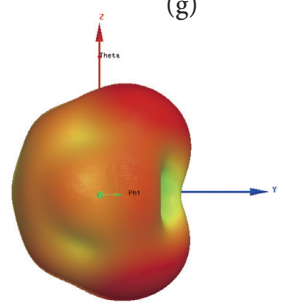

(j)

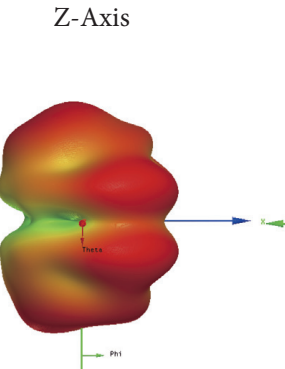

(b)

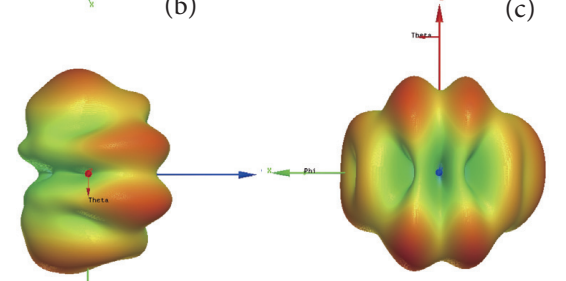

(e)

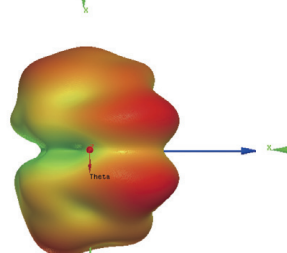

(h)

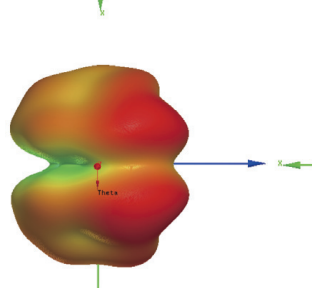

(k) (f)

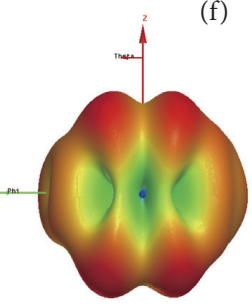

(i)

(c)

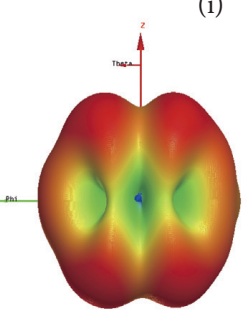

(l)
Total gain $[\mathrm{dB}]$

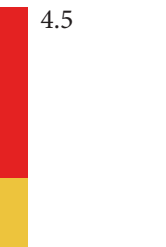

FIgURE 7: Simulated 3D radiation patterns when both Port1 and Port2 are fed on the (a) $x$-axis, (b) $y$-axis, and (c) $z$-axis for the base antenna at $3.60 \mathrm{GHz}$; on the (d) $x$-axis, (e) $y$-axis, and (f) $z$-axis for Ant. 1 at $3.55 \mathrm{GHz}$; on the (g) $x$-axis, (h) $y$-axis, and (i) $z$-axis for Ant. 2 at $3.70 \mathrm{GHz}$; and, finally, on the (j) $x$-axis, (k) $y$-axis, and (l) $z$-axis for Ant. 3 at $3.34 \mathrm{GHz}$. 

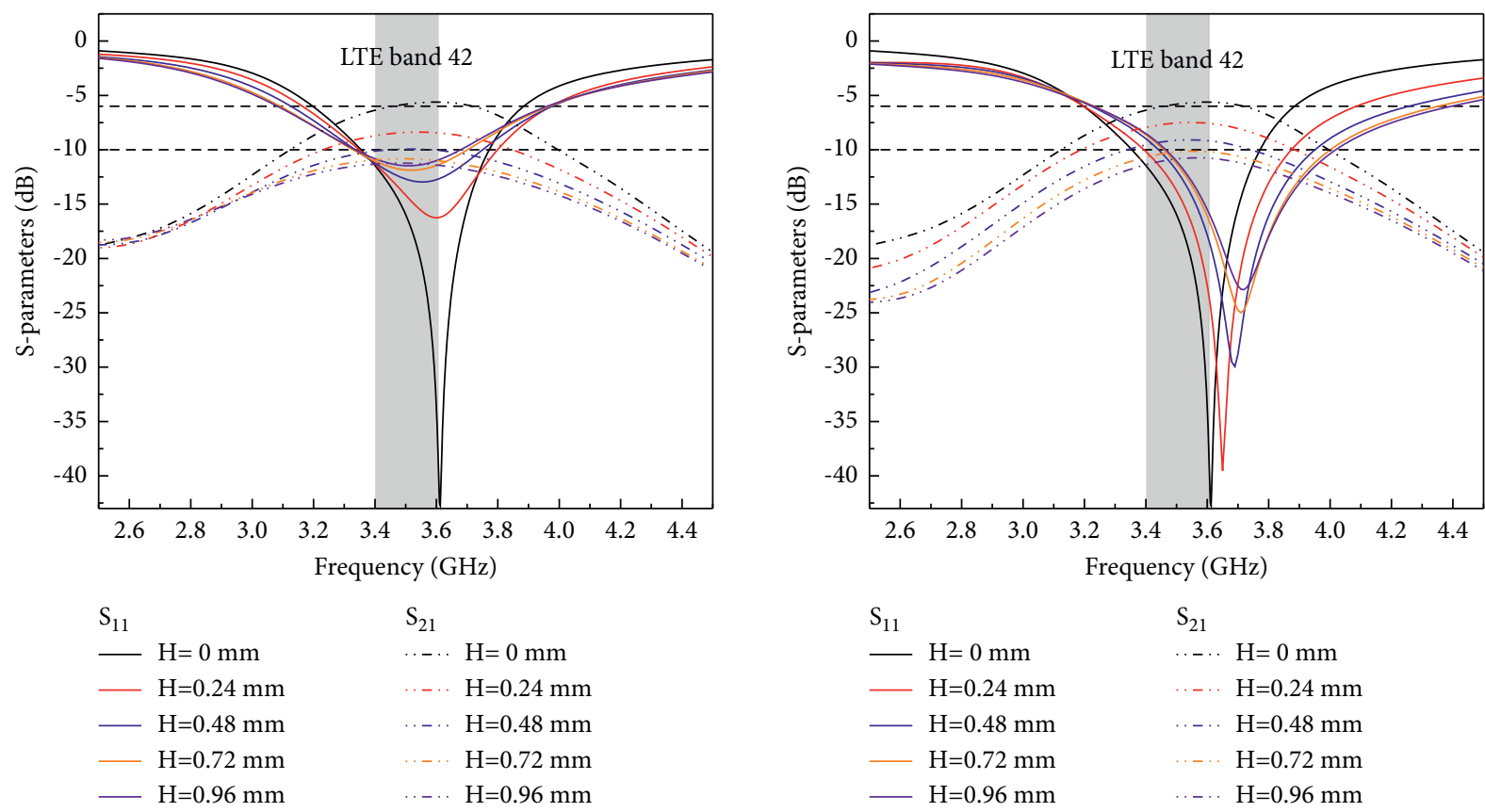

(a)
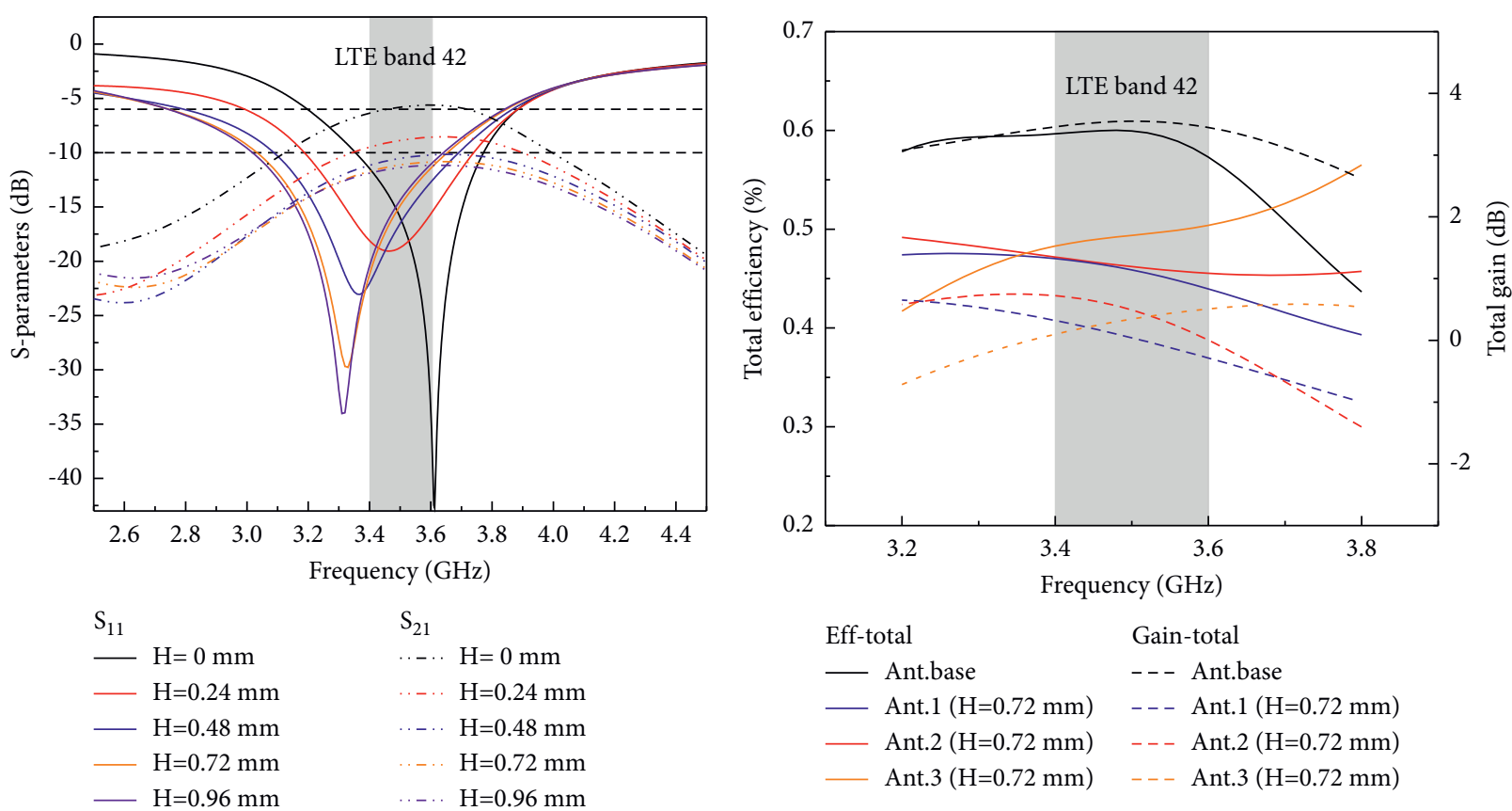

(c)

(d)

Figure 8: Simulated S-parameters with different ferrite-film thicknesses for (a) Ant.1, (b) Ant.2, and (c) Ant.3. (d) Simulated total efficiencies and gains of the antenna without and with a $0.72 \mathrm{~mm}$ thickness ferrite-film loading.

a slight shift of resonant frequency. On the other hand, with the ferrite film on Area2 and Area3, the great resonant frequency shifts of $S_{11}$ can be observed by the increase of the ferrite-film thickness, in which upward for Ant.2 and downward for Ant.3. Figure 8(d) shows the simulated total efficiencies and gains of three antennas by fixing the film thickness as $H=0.72 \mathrm{~mm}$. The ferrite-film loading will sacrifice the efficiency and gain of the antenna at a certain extent, but it can be controlled by choosing a proper film thickness and loading area, as that Ant.3 shows a total efficiency of up to $50 \%$ and a gain of up to $0.5 \mathrm{~dB}$.

\section{Experimental Verification}

To verify the inhibitory effect of magnetic materials in terms of multiantenna coupling, we prepared the base antenna designed in Figure 1 and used commercial NiZn-ferrite films 


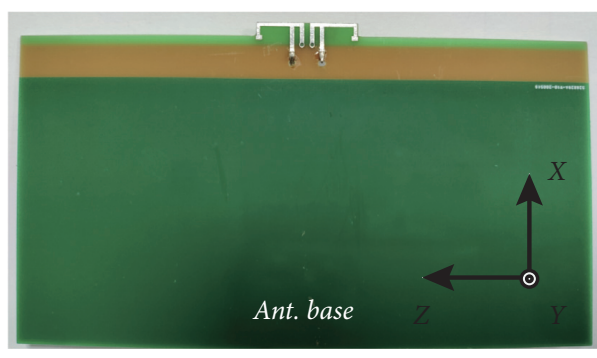

(a)

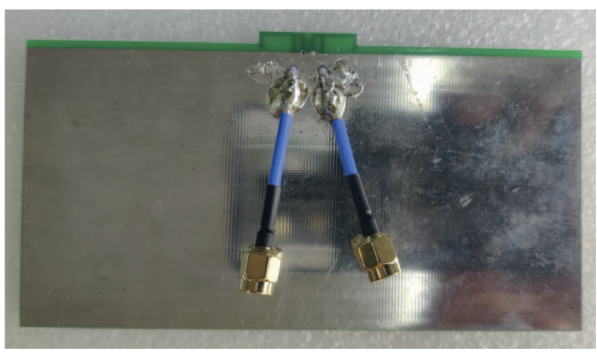

(b)

FIGURE 9: Photographs of the fabricated base antenna in (a) top view and (b) bottom view.

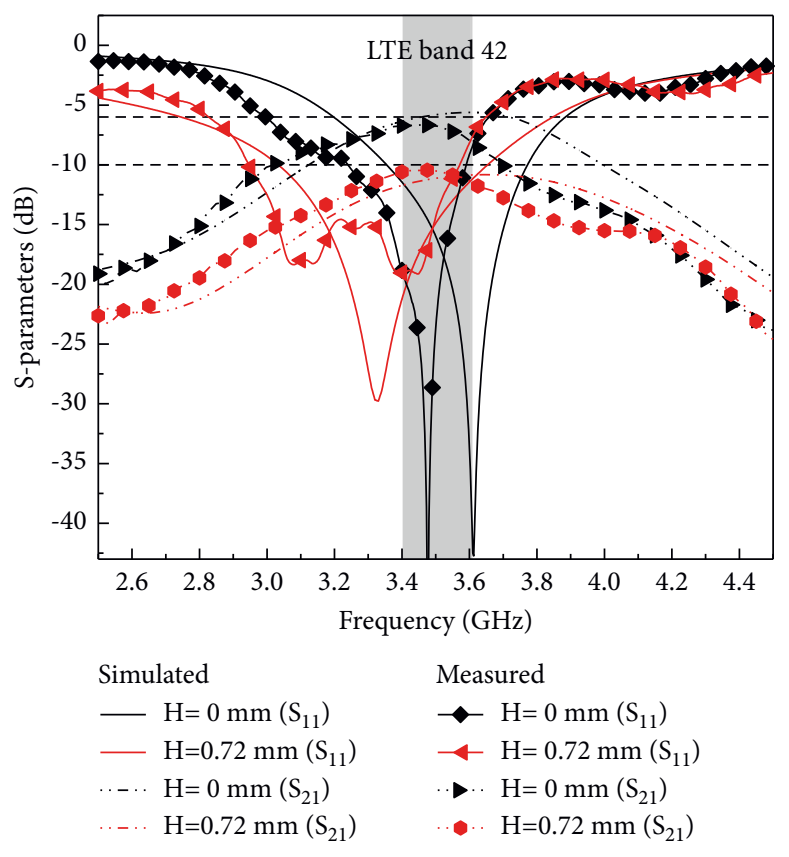

(a)
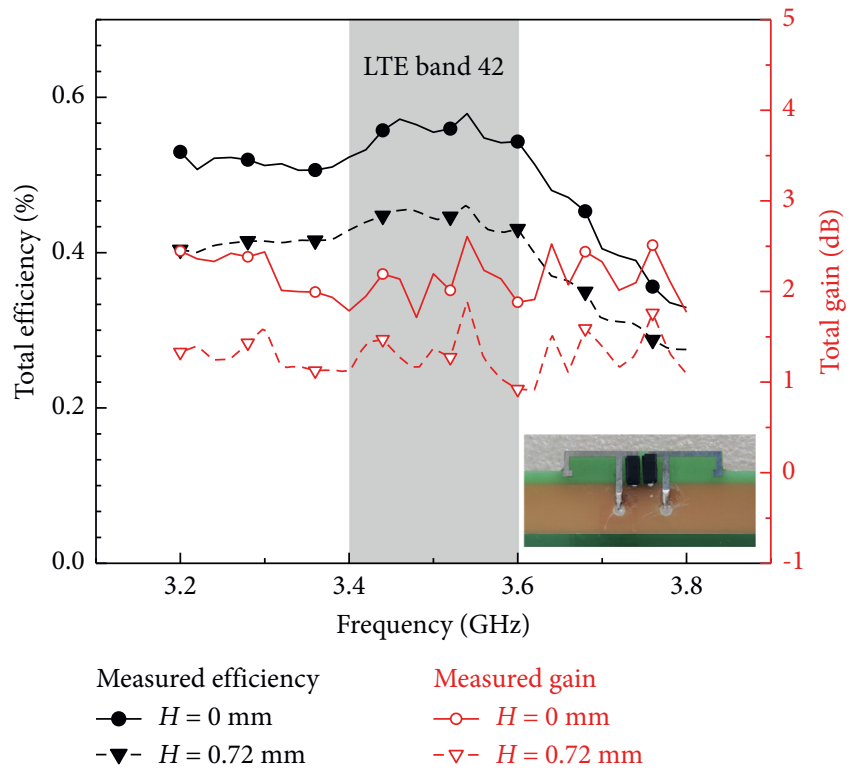

(b)

Figure 10: (a) Simulated and measured $S$-parameters and (b) measured total efficiencies and total gains for Ant.3 without and with a $0.72 \mathrm{~mm}$ thickness ferrite-film loading.

(PHF15010, Shenzhen PH Functional Materials Co., Ltd., China) with a thickness of $0.06 \mathrm{~mm}$ as the loading material. Figure 9 shows photographs of the fabricated dual-element antennas. The front portion is the radiation area, and the top part is the designed dual-unit antenna, shown in Figure 9(a). The back portion represents the ground area, shown in Figure 9(b). Each patch was printed on an FR-4 substrate and fed by two coaxial cables.

By considering the simulated S-parameter and total efficiency results, we take Ant. 3 and load it with a $0.72 \mathrm{~mm}$ thickness ferrite film as an optimum example to verify the radiation performances of the proposed ferrite-film loading antenna. We tested the $S$-parameter and total efficiency and gain of the antenna both with and without ferrite-film loading in the experiment. The simulated and measured $S$-parameters of the monopole pair without and with the decoupling component are compared in Figure 10(a). When the base antenna is not loaded with a ferrite film, the mutual coupling of $S_{21}$ between the two monopole elements is more than
$-10 \mathrm{~dB}$ at the frequency band of $3.4-3.6 \mathrm{GHz}$; in the case where the antenna had ferrite-film loading, the mutual coupling is significantly reduced to be lower than $-10 \mathrm{~dB}$ over the entire LTE Band 42 . The measured results are well consistent with the simulated results. In addition, the $-6 \mathrm{~dB}$ bandwidth of the base antenna can be widened by the ferritefilm loading from $3.19-3.90 \mathrm{GHz}$ to $2.74-3.8 \mathrm{GHz}$ in the simulation, as well as the measured bandwidth from $3.20-3.88 \mathrm{GHz}$ to $2.83-3.65 \mathrm{GHz}$. The measured total efficiencies and gains of the proposed antenna, when unloading and loading the ferrite film, are also presented in Figure 10(b), at $3.4 \mathrm{GHz}$. It can be seen that the total efficiency and gain of the antenna decrease slightly after the ferrite film covers them and the total efficiency at the target $5 \mathrm{G}$ band of LTE Band 42 $(3.4 \mathrm{GHz}-3.6 \mathrm{GHz})$ is over $45 \%$, as well as a $1.7 \mathrm{~dB}$ gain. On the other hand, the measured radiation patterns of the antenna with ferrite-film loading in the $E$-plane and $H$-plane, shown in Figure 11, exhibit no changes in comparison to the base antenna. Furthermore, we also compare several 

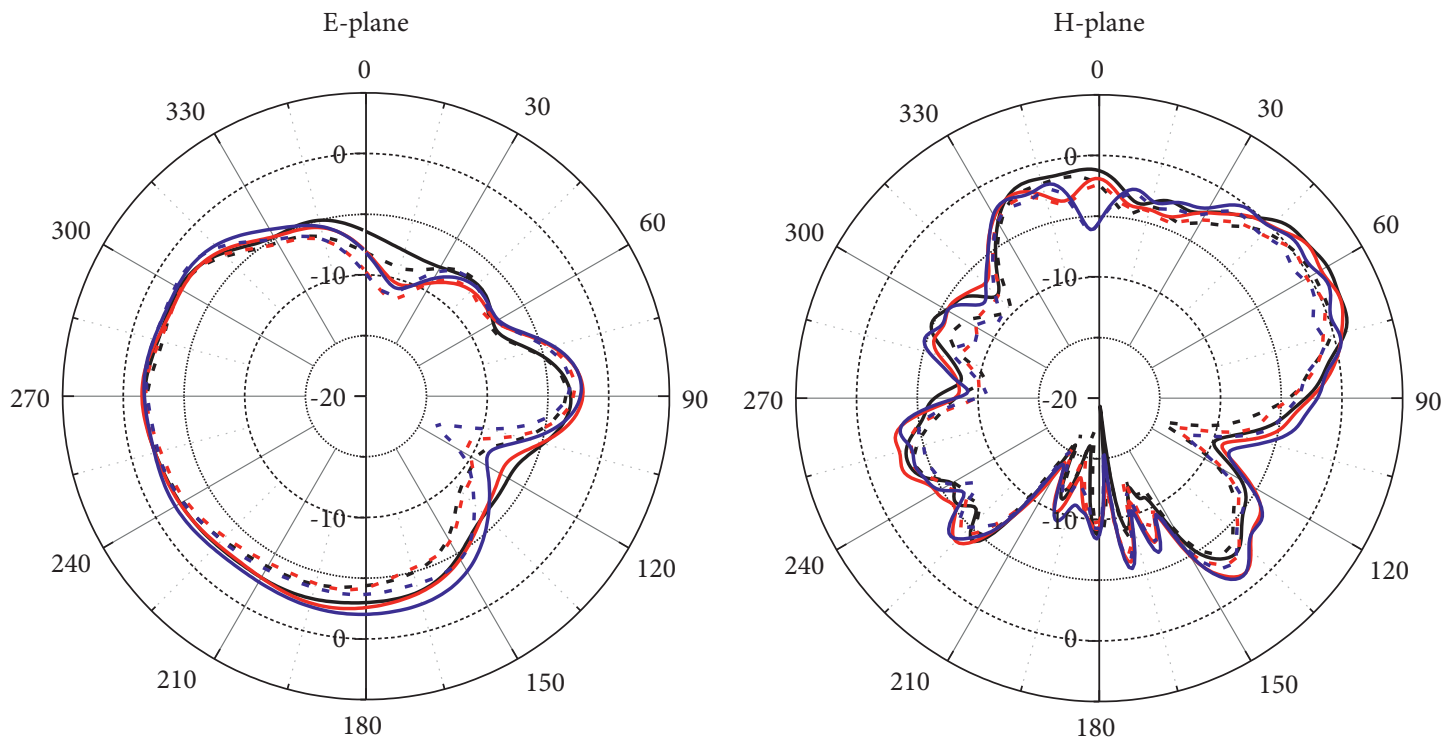

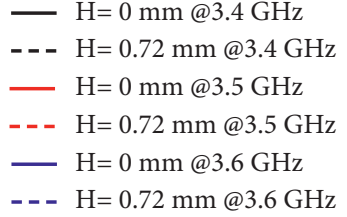

(a)

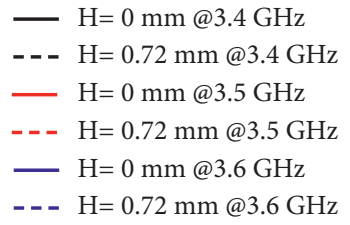

(b)

Figure 11: Measured radiation patterns at $3.4 \mathrm{GHz}, 3.5 \mathrm{GHz}$, and $3.6 \mathrm{GHz}$ of (a) E-plane and (b) $H$-plane for Ant. 3 without and with a $0.72 \mathrm{~mm}$ thickness ferrite-film loading.

TABLE 2: Comparison of the proposed antenna with existing works.

\begin{tabular}{|c|c|c|c|c|c|c|}
\hline Ref. & $\begin{array}{c}\text { Element size } \\
\left(L \times W \times H \mathrm{~mm}^{3}\right)\end{array}$ & Method & Bandwidth & Isolation $(\mathrm{dB})$ & Element distance $(\mathrm{mm})$ & Efficiency \\
\hline [8] & $16 \times 5 \times 1$ & Decoupling-loop & $8.9 \%(3.33-3.65 \mathrm{GHz})$ & 13 & 1 & $65-80 \%$ \\
\hline$[11]$ & $11 \times 43.5 \times 1$ & Tapered-slot-etched method & $40.8 \%(1.7-2.7 \mathrm{GHz})$ & 40 & 8.5 & - \\
\hline$[12]$ & $28 \times 1 \times 29$ & Parasitic structure & $5 \%(2.33-2.45 \mathrm{GHz})$ & 25 & 28 & $72-83 \%$ \\
\hline [16] & $6.7 \times 15.5 \times 3.08$ & Self-neutralized technique & $4.1 \%(4.8-5.0 \mathrm{GHz})$ & 23 & 3.2 & $78-80 \%$ \\
\hline [17] & $27.3 \times 27.3 \times 3.175$ & Weak field & $5.7 \%(3.4-3.6 \mathrm{GHz})$ & 12.0 & 6.5 & - \\
\hline$[21]$ & $35 \times 45 \times 1.6$ & Metamaterial & $8.8 \%(9.12-9.96 \mathrm{GHz})$ & 10 & 20 & - \\
\hline [37] & $20 \times 32 \times 1.6$ & Extra branch & $14.3 \%(3.3-3.8 \mathrm{GHz})$ & 14 & 2 & $68-80 \%$ \\
\hline [39] & $9.6 \times 3 \times 0.8$ & Lumped components & $3.9 \%(3.42-3.59 \mathrm{GHz})$ & 13.5 & 1 & $32-51 \%$ \\
\hline This work & $11.3 \times 4 \times 1$ & Ferrite loading & $9.1 \%(3.35-3.67 \mathrm{GHz})$ & 10.43 & 1.4 & $43-45.5 \%$ \\
\hline
\end{tabular}

important parameters that reflect the performances of the MIMO systems with previous works in Table 2. The MIMO antennas can be decoupled with higher isolations and higher efficiencies by choosing the design methodologies adequately, such as tapered-slot-etched method, parasitic structure, selfneutralized technique, and extra branch, but the element sizes and distances are much larger; on the other hand, the antennas can be designed with smaller sizes, but the isolations are always around $10 \mathrm{~dB}$ and they exhibit limited efficiencies and bandwidths; otherwise, the decoupling techniques with metamaterial and ferrite loading can effectively repress the mutual coupling and increase the working frequency band while keeping constant the physical dimensions. A compromise must be made among the performance parameters, element size, and design complexity to drive the different decoupling techniques' application in the MIMO systems. Here, it can be derived that, by comparison with other typical decoupling techniques, the ferrite-film loading decoupling can be used in a smaller size MIMO antenna element with a closer element distance and high isolation, showing better operating performance with enhanced bandwidth; its efficiency can reach $45.5 \%$. This comparison further confirms that coupled antennas with ferrite-film decoupling can be applied in the field of 5G MIMO.

\section{Conclusion}

In this paper, we propose a method to mitigate the mutual coupling between MIMO antennas by using ferrite-film loading. By selectively covering ferrite on the radiation 
surfaces of the antenna, the induced field can be suppressed by the EM field interactions with the ferrite film, which leads to the decoupling process in the MIMO antenna. A two-IFAelement MIMO antenna operating in LTE Band 42 $(3.4-3.6 \mathrm{GHz})$ has been designed and fabricated to utilize this strategy. Through an optimization process using simulations and experimental investigations, the MIMO antenna with a covering of ferrite film on the grounding branch of the IFA element can be decoupled adequately with high isolation and exhibits the best operating performance with enhanced bandwidth; its efficiency can reach $45 \%$. A physical model has also been established to interpret the operating mechanism of ferrite-film loading on the decoupling process of the IFAs. This decoupling technique, which involves simply loading a ferrite film on a small radiation area of the antenna, could enhance the applicability and flexibility of the MIMO antennas, allowing them to modify their operating characteristics depending on channel conditions. This study provides an alternative method for developing multiantenna-based MIMO mobile platforms and has both practical and theoretical significance.

\section{Data Availability}

The experimental data used to support the findings of this study are available from the corresponding author upon request.

\section{Conflicts of Interest}

The authors declare that they have no conflicts of interest.

\section{Authors' Contributions}

D. Wu and Y. Qiu contributed equally to this work.

\section{Acknowledgments}

This study was supported by the funds of the National Natural Science Foundation of China under Grant nos. 11972333, 11902316, and 51902300, the Natural Science Foundation of Zhejiang Province under Grant nos. LZ19A020001, LQ19F010005, and LY21F010011, and the Open Project Funding of the Key Laboratory of Electromagnetic Wave Information Technology and Metrology of Zhejiang Province under Grants 2019KF0002 and 2020KF0002. The antenna's efficiency, gain, and pattern were measured in Hangzhou RFID Center, CAS. The authors would like to express their sincere appreciation to these supports.

\section{References}

[1] J. Huang, C. X. Wang, H. Chang, J. Sun, and X. Gao, "Multifrequency multi-scenario millimeter wave MIMO channel measurements and modeling for B5G wireless communication systems," IEEE Journal on Selected Areas in Communications, vol. 38, no. 9, pp. 2010-2025, 2020.

[2] R. Chataut and R. Akl, "Massive MIMO systems for 5G and beyond networks-overview, recent trends, challenges, and future research direction," sensors (Basel), vol. 20, no. 10, 2020.
[3] M. Alibakhshikenari, B. S. Virdee, L. Azpilicueta et al., "A comprehensive survey of "metamaterial transmission-line based antennas: design, challenges, and applications"," IEEE Access, vol. 8, pp. 144778-144808, 2020.

[4] W. Zhou, L. Huang, B. Luo, R. Mai, Z. He, and A. P. Hu, “A general mutual coupling model of MIMO capacitive coupling interface with arbitrary number of ports," IEEE Transactions on Power Electronics, vol. 36, no. 6, pp. 6163-6167, 2021.

[5] Z. Zhang, Antenna Design for mobile Devices, John Wiley \& Sons, Hoboken, NJ, USA, 2017.

[6] Y. Wang and Z. Du, "A wideband printed dual-antenna system with a novel neutralization line for mobile terminals," IEEE Antennas and Wireless Propagation Letters, vol. 12, pp. 1428-1431, 2013.

[7] M. Li, L. Jiang, and K. L. Yeung, "A general and systematic method to design neutralization lines for isolation enhancement in MIMO antenna arrays," IEEE Transactions on Vehicular Technology, vol. 69, no. 6, pp. 6242-6253, 2020.

[8] H. Piao, Y. Jin, Y. Xu, and L. Qu, "MIMO ground-radiation antennas using a novel closed-decoupling-loop for $5 \mathrm{G}$ applications," IEEE Access, vol. 8, pp. 142714-142724, 2020.

[9] S. Xiao, M. C. Tang, Y. Y. Bai, and S. Gao, "Mutual coupling suppression in microstrip array using defected ground structure," IET Microwaves, Antennas \& Propagation, vol. 5, no. 12, 2011.

[10] X. Tan, W. Wang, Y. Wu, Y. Liu, and A. A. Kishk, "Enhancing isolation in dual-band meander-line multiple antenna by employing split EBG structure," IEEE Transactions on Antennas and Propagation, vol. 67, no. 4, pp. 2769-2774, 2019.

[11] S. Tebache, A. Belouchrani, F. Ghanem, and A. Mansoul, "Novel reliable and practical decoupling mechanism for strongly coupled antenna arrays," IEEE Transactions on Antennas and Propagation, vol. 67, no. 9, pp. 5892-5899, 2019.

[12] M. Li and S. Cheung, "A novel calculation-based parasitic decoupling technique for increasing isolation in multipleelement MIMO antenna arrays," IEEE Transactions on Vehicular Technology, vol. 70, no. 1, pp. 446-458, 2021.

[13] K. Ding, C. Gao, D. Qu, and Q. Yin, "Compact broadband MIMO antenna with parasitic strip," IEEE Antennas and Wireless Propagation Letters, vol. 16, pp. 2349-2353, 2017.

[14] F. Amin, R. Saleem, T. Shabbir, and S. U. Rehman, "A compact quad-element UWB-MIMO antenna system with parasitic decoupling mechanism," applied sciences, vol. 9, no. 11, 2019.

[15] L. Sun, Y. Li, Z. Zhang, and H. Wang, "Self-decoupled MIMO antenna pair with shared radiator for $5 \mathrm{G}$ smartphones," IEEE Transactions on Antennas and Propagation, vol. 68, no. 5, pp. 3423-3432, 2020.

[16] C.-Z. Han, L. Xiao, Z. Chen, and T. Yuan, "Co-located selfneutralized handset antenna pairs with complementary radiation patterns for 5G MIMO applications," IEEE Access, vol. 8, pp. 73151-73163, 2020.

[17] H. Lin, Q. Chen, Y. Ji, X. Yang, J. Wang, and L. Ge, "Weakfield-based self-decoupling patch antennas," IEEE Transactions on Antennas and Propagation, vol. 68, no. 6, pp. 4208-4217, 2020.

[18] X. Zhao, S. P. Yeo, and L. C. Ong, "Decoupling of inverted-F antennas with high-order modes of ground plane for $5 \mathrm{G}$ mobile MIMO platform," IEEE Transactions on Antennas and Propagation, vol. 66, no. 9, pp. 4485-4495, 2018.

[19] C. Segura-Gomez, A. Palomares-Caballero, A. Alex-Amor, J. Valenzuela-Valdes, and P. Padilla, "Modular design for a stacked SIW antenna array at ka-band," IEEE Access, vol. 8, pp. 158568-158578, 2020. 
[20] M. Alibakhshikenari, F. Babaeian, B. S. Virdee et al., "A comprehensive survey on "various decoupling mechanisms with focus on metamaterial and metasurface principles applicable to SAR and MIMO antenna systems"," IEEE Access, vol. 8, pp. 192965-193004, 2020.

[21] M. Alibakhshikenari, M. Khalily, B. S. Virdee, C. H. See, R. A. Abd-Alhameed, and E. Limiti, "Mutual-coupling isolation using embedded metamaterial EM bandgap decoupling slab for densely packed array antennas," IEEE Access, vol. 7, pp. 51827-51840, 2019.

[22] E. K. I. Hamad and M. Z. M. Hamdalla, "Design of miniaturized and high isolation metamaterial-based mimo antenna for mobile terminals," JES. Journal of Engineering Sciences, vol. 45, no. 6, pp. 763-772, 2017.

[23] E. Andreou, T. Zervos, A. A. Alexandridis, and G. Fikioris, "Magnetodielectric materials in antenna design: exploring the potentials for reconfigurability," IEEE Antennas and Propagation Magazine, vol. 61, no. 1, pp. 29-40, 2019.

[24] J. Lee, Y. K Hong, S. Bae, G. S. Abo, W. M. Seong, and G. H. Kim, "Miniature long-term evolution (LTE) MIMO ferrite antenna," IEEE Antennas and Wireless Propagation Letters, vol. 10, pp. 603-606, 2011.

[25] M. I. Kitra, C. J. Panagamuwa, P. McEvoy, J. C. Vardaxoglou, and J. R. James, "Low SAR ferrite handset antenna design," IEEE Transactions on Antennas and Propagation, vol. 55, no. 4, pp. 1155-1164, 2007.

[26] R. V. Petrov, A. S. Tatarenko, G. Srinivasan, and J. V. Mantese, "Antenna miniaturization with ferrite ferroelectric composites," Microwave and Optical Technology Letters, vol. 50, no. 12, pp. 3154-3157, 2008.

[27] Y. Guo-Min Yang, X. Xing, A. Daigle et al., "Electronically tunable miniaturized antennas on magnetoelectric substrates with enhanced performance," IEEE Transactions on Magnetics, vol. 44, no. 11, pp. 3091-3094, 2008.

[28] Y. Guo-Min Yang, A. Shrabstein, X. Xing et al., "Miniaturized antennas and planar bandpass filters with self-biased NiCoferrite films," IEEE Transactions on Magnetics, vol. 45, no. 10, pp. 4191-4194, 2009.

[29] Y. Guo-Min Yang, X. Xing, A. Daigle et al., "Tunable miniaturized patch antennas with self-biased multilayer magnetic films," IEEE Transactions on Antennas and Propagation, vol. 57, no. 7, pp. 2190-2193, 2009.

[30] A. Belous, O. Fedorchuk, S. Solopan, P. Maksym, and Z. Igor, "Magnetically tunable composite ferrite-dielectric microwave elements," Journal of Magnetism and Magnetic Materials, vol. 505, 2020.

[31] K. K. Jain and P. Kishan, "Microwave ferrite devices," IEEE Technical Review, vol. 14, no. 6, pp. 373-386, 2015.

[32] A. Nafe and A. Shamim, "An integrable SIW phase shifter in a partially magnetized ferrite LTCC package," IEEE Transactions on Microwave Theory and Techniques, vol. 63, no. 7, pp. 2264-2274, 2015.

[33] F. A. Ghaffar, J. R. Bray, M. Vaseem, L. Roy, and A. Shamim, "Theory and design of tunable full-mode and half-mode ferrite waveguide isolators," IEEE Transactions on Magnetics, vol. 55, no. 8, pp. 1-8, 2019.

[34] C. Fournet-Fayas, A. C. Priou, and G. E. Forterre, "A 50-kW CW ferrite circulator in S band," IEEE Transactions on Microwave Theory and Techniques, vol. 26, no. 5, pp. 360-363, 1978.

[35] J. Diao, L. Liu, and K. F. Warnick, "An intuitive way to understand mutual coupling effects in antenna arrays using the poynting streamline method," IEEE Transactions on Antennas and Propagation, vol. 67, no. 2, pp. 884-891, 2019.
[36] A. H. Robbins and W. Miller, Circuit analysis: Theory and practice: Nelson Education, 2012.

[37] J. Sui and K.-L. Wu, "A self-decoupled antenna array using inductive and capacitive couplings cancellation," IEEE Transactions on Antennas and Propagation, vol. 68, no. 7, pp. 5289-5296, 2020.

[38] T. Wu, G. Yu, J. Pang et al., "The low-frequency improvement with loading soft magnetic ferrite films for multiband antenna applications," International Journal of Applied Electromagnetics and Mechanics, vol. 66, no. 2, pp. 359-368, 2021.

[39] C. Deng, D. Liu, and X. Lv, "Tightly arranged four-element MIMO antennas for $5 \mathrm{G}$ mobile terminals," IEEE Transactions on Antennas and Propagation, vol. 67, no. 10, pp. 6353-6361, 2019. 